\title{
An analytic model for the galactic winds and mass outflows
}

\author{
Chenggang Shu ${ }^{1,2,4}$, H.J. $\mathrm{Mo}^{2}$, Shude $\mathrm{Mao}^{3} \star$ \\ 1 Shanghai Astronomical Observatory, Chinese Academy of Sciences, Shanghai 200030, China \\ 2 Max-Planck-Institut für Astrophysik, Karl-Schwarzschild-Strasse 1, Postfach 1317 D-85741 Garching, Germany \\ 3 Jodrell Bank Observatory, Univ. of Manchester, Macclesfield, Cheshire SK11 9DL, UK \\ 4 The Joint Lab of Optical Astronomy, Chinese Academy of Sciences \\ Accepted ....... Received ......; in original form ......
}

\begin{abstract}
Galactic winds and mass outflows are observed both in nearby starburst galaxies and in high-redshift star-forming galaxies. In this paper we develop a simple analytic model to understand the observed superwind phenomenon. Our model is built upon the model of McKee \& Ostriker (1977) for the interstellar medium. It allows one to predict how properties of a superwind, such as wind velocity and mass outflow rate, are related to properties of its starforming host galaxy, such as size, gas density and star formation rate. The model predicts a threshold of star formation rate density for the generation of observable galactic winds. Galaxies with more concentrated star formation produce superwinds with higher velocities. The predicted mass outflow rates are comparable to (or slightly larger than) the corresponding star formation rates. We apply our model to both local starburst galaxies and high-redshift Lyman break galaxies, and find its predictions to be in good agreement with current observations. Our model is simple, and so can be easily incorporated into numerical simulations and semi-analytical models of galaxy formation.
\end{abstract}

Key words: galaxies: star formation - galaxies: kinematics and dynamics galaxies: superwind and outflow

\footnotetext{
^ E-mail: cgshu@center.shao.ac.cn, hom@mpa-garching.mpg.de, smao@jb.man.ac.uk
} 


\section{INTRODUCTION}

Galactic-scale bulk motions of gas, such as galactic winds and mass outflows, have been observed both in local starburst galaxies (Forbes et al 2000; Heckman et al 2000 and references therein) and in high redshift galaxies (Pettini et al 2000, 2001; Dawson et al 2002; Adelberger et al 2002). Evidence for such superwinds and mass outflows comes from X-ray emission produced by the hot gas or optical line emission from the warm gas associated with star-forming galaxies (Martin, Kobulnicky \& Heckman 2002; Strickland et al 2002). Based on mediumresolution spectra of $\mathrm{Na} \mathrm{D}(\lambda \lambda 5890,5896)$ and X-ray observations of some local starburst galaxies, Heckman et al (2000) constrained wind velocities in the range $\sim 400-800 \mathrm{~km} \mathrm{~s}^{-1}$ and mass outflow rates comparable to star formation rates (hereafter SFRs). Using observations of nebular absorption lines and Ly- $\alpha$ emission lines, Pettini et al (2000) and Adelberger et al (2002) showed that galactic winds are common in high-redshift Lyman break galaxies. The inferred wind velocities for these objects range from several hundred $\mathrm{km} \mathrm{s}^{-1}$ to about $1000 \mathrm{~km} \mathrm{~s}^{-1}$, with a median value of about $500 \mathrm{~km} \mathrm{~s}^{-1}$. For the bright gravitationally lensed Lyman-break galaxy, MS 1512-cB52 ( $z=2.72)$, Pettini et al $(2000,2001)$ estimated the mass outflow rate to be $\sim 60 \mathrm{M}_{\odot} \mathrm{yr}^{-1}$, comparable to the star formation rate of this system derived from its UV luminosity.

These bulk motions of gas (superwinds) in star-forming galaxies are believed to be driven by the kinetic energy from supernova (hereafter SN) explosions, and may change the thermal and chemical properties of the intergalactic medium (IGM) that forms galaxies (Ferrara, Pettini \& Schekinov 2000; Furlanetto \& Loeb 2002). It is therefore essential to have a proper understanding of these phenomena in order to understand galaxy formation itself.

So far, our understanding of galactic superwinds is quite incomplete. Based on the SN remnant evolution model of McKee \& Ostriker (1977), Efstathiou (2000) established a multiphase interstellar medium (ISM) model in which galactic winds result from the expansion of hot phase gas. Combined with other physical prescriptions such as the infall of the cooling gas and star formation, he investigated the formation and evolution of disk galaxies in this model, and studied the dependence of mass outflow from a galaxy on the circular velocity of its host halo. Silk $(2001,2002)$ proposed an analytic model for the feedback process and outflow in galaxies based on disk gravitational instability and multiphase ISM. Numerically there have also been attempts to use simulations to understand how galactic winds can be

produced (e.g. Tomisaka \& Bregman 1993; Suchkov et al 1994; Mac Low \& Ferrara 1999).

(C) 2002 RAS, MNRAS 000, 000-000 
However, current simulations still lack the resolution to deal with processes such as the evolution of supernova remnants and the formation of multi-phase gas that may be essential for the formation of superwinds. Because of this, simple assumptions are usually made about superwinds when studying their impact on the IGM (e.g. Strickland \& Stevens 2000; Scannapieco, Ferrara \& Broadhurst 2000; Scannapieco, Ferrara \& Madau 2002; Theuns et al 2002; White, Hernquist \& Springel 2002).

In this paper, we attempt to construct a simple analytic model for superwinds. Our model is based on the model of McKee \& Ostriker (1977) for supernova evolution in the ISM. Our goal is to understand how properties of superwinds, such as wind velocity and mass outflow rate, are related to the properties of star-forming galaxies, such as size, gas density and SFR. The establishment of such relations will allow one to include superwinds in simulations and semi-analytical models of galaxy formation. We test our model by comparing its predictions with observations of superwinds in local starburst galaxies and in high-redshift Lyman-break galaxies.

Our basic model for the generation of superwinds is similar to that of Efstathiou (2000), as both his paper and ours are based on McKee \& Ostriker (1977). While Efstathiou focused on the details about the assembly and star formation in two example galaxies (one dwarftype and the other Milky-Way type), we focus on the statistical properties of superwinds in star forming galaxies based on simple empirical models about star formation. In addition, we compare our theoretical predictions with observations for local starburst galaxies and high-redshift Lyman-break galaxies.

\section{MODEL}

In this section, we will use the model of McKee \& Ostriker (1977, hereafter MO77) for supernova evolution in the interstellar medium to construct a model of galactic winds and mass outflows for star forming galaxies. Before going into the details, we summarize here the basic idea in our modeling (see also Efstathiou 2000, hereafter E2000, for more details).

In the model of MO77, cold star-forming gas in the ISM is assumed to be in the form of cold clouds surrounded by the warm ISM with temperature of about $\lesssim 10^{4} \mathrm{~K}$. Massive stars evolve and explode in a few million years as supernovae (SNe), and SN remnants propagate to form a low-density hot medium with a temperature of about $10^{5-6} \mathrm{~K}$. The expansion of the SN remnants compresses and sweeps out material from the cold clouds in the shock front to 
produce large-scale bulk motions in the ISM, which can be accelerated by the pressure in the SN remnants. We assume such bulk motion to be responsible for the observed superwinds and mass outflows. In such a scenario, the properties of the superwind from a galaxy are expected to depend not only on the properties of the ISM, but also on other properties of the galaxy in consideration. In what follows, we quantify the main processes involved in our model.

\subsection{Supernovae evolution, interstellar media and galactic winds}

Star formation generally takes place in giant molecular clouds which consist of many small and dense clouds. The differential number density distribution of clouds as a function of cloud radius is assumed to follow a power-law (MO77, E2000)

$\frac{d N_{c}}{d a}=n_{0} a^{-4}, \quad a_{1} \leq a \leq a_{\mathrm{u}}$,

where $a_{1}$ and $a_{\mathrm{u}}$ are lower and upper limits for the cloud radii, and $n_{0}$ is a normalization constant. We take, following MO77,

$a_{\mathrm{l}}=0.5 \mathrm{pc}, \quad a_{\mathrm{u}} / a_{\mathrm{l}}=20$.

We caution that the exact value of the lower limit radius $\left(a_{1}\right)$ is unclear. It is generally believed to be in the range of 0.5 to $1 \mathrm{pc}$ (MO77); recent observations by Olmi \& Testi (2002) suggested that $a_{1} \sim 0.55 \mathrm{pc}$.

It is easy to show by integration that the local cloud number density, $n_{\mathrm{cl}}$, is related to $n_{0}$ by

$n_{\mathrm{cl}}=n_{0} / 3 a_{1}^{3}$, for $a_{\mathrm{u}} \gg a_{1}$.

Assuming that $\bar{\rho}_{\mathrm{c}}$ is the mean mass density within individual clouds, the mean cold gas density $\bar{\rho}_{\text {cold }}$ can be written as

$\bar{\rho}_{\text {cold }}=\int_{a_{1}}^{a_{\mathrm{u}}} \frac{d N_{c}}{d a} \frac{4 \pi}{3} a^{3} \bar{\rho}_{\mathrm{c}} d a=\frac{4 \pi}{3} \bar{\rho}_{\mathrm{c}} n_{0} \ln \frac{a_{\mathrm{u}}}{a_{\mathrm{l}}}$.

Thus, the volume filling factor of the cold gas is

$f_{\mathrm{c}}=\frac{\bar{\rho}_{\text {cold }}}{\bar{\rho}_{\mathrm{c}}}$.

The porosity $P$ is related to $f_{\mathrm{c}}$ by $f_{\mathrm{c}} \equiv e^{-P}$.

According to MO77, an expanding SN remnant will sweep up the ambient cold gas with a total mass of

$M_{\mathrm{ev}}=540 \mathrm{E}_{51}{ }^{6 / 5} n_{\mathrm{h}}^{-4 / 5} \Sigma^{-3 / 5} \mathrm{M}_{\odot}$ 
where $\mathrm{E}_{51}$ is the energy output by one $\mathrm{SN}$ in unit of $10^{51} \mathrm{erg}, n_{\mathrm{h}}$ is the hot gas density interior to the SN remnant and $\Sigma$ is the evaporation parameter

$\Sigma=\frac{\gamma}{4 \pi a_{1} n_{\mathrm{cl}} \phi_{k}}=\frac{\gamma a_{1}^{2} \ln \left(a_{\mathrm{u}} / a_{\mathrm{l}}\right)}{\phi_{k}} \frac{\bar{\rho}_{\mathrm{c}}}{\bar{\rho}_{\mathrm{cold}}}$.

Here $\gamma$ is the ratio of the blast wave velocity to the isothermal sound speed of the hot phase, which is usually taken to be 2.5 (E2000), and the parameter $\phi_{k}$ denotes the efficiency of conduction relative to the classic thermal conductivity of the clouds. $\phi_{k}$ is roughly in the range of 0.1 to 0.01 due to the suppression of heat conduction by tangled magnetic fields, turbulence, etc (E2000).

As argued by several authors (Silk 1997, 2001, 2002; E2000; Clarke \& Oey 2002), star formation in galaxies may proceed in such a way that the porosity $P$ is always maintained as a constant value close to unity in star formation regions. This constancy in the porosity $P$ is supported by observations in the Milky Way, and can be physically understood as follows. If the porosity were too large, the cold gas fraction would be small and the star formation activity would be reduced. The hot gas will then cool quickly, and hence leading to a lower porosity. Conversely, if the porosity were too small, the cold gas fraction would be large. As the dynamical timescale is likely to be short in the star formation regions, star formation will ensue and hence lead to a higher SFR and higher porosity. In the present study, we assume the porosity to be unity everywhere in a star formation region.

Adopting $a_{\mathrm{u}} / a_{\mathrm{l}}=20$, we can rewrite the evaporation parameter $(\Sigma)$ as

$\Sigma=752 f_{\mathrm{c}}^{-1}\left(\frac{\gamma}{2.5}\right)\left(\frac{\phi_{k}}{0.01}\right)^{-1}\left(\frac{a_{1}}{\mathrm{pc}}\right)^{2} \mathrm{pc}^{2}$.

Notice that the value of $\Sigma$ is not very sensitive to the ratio $a_{\mathrm{u}} / a_{1}$ because it enters eq. (8) logarithmically. For convenience, we define a new parameter

$f_{\Sigma}=\frac{\Sigma}{\Sigma_{\odot}}, \quad \Sigma_{\odot}=95 \mathrm{pc}^{2}$

which is the evaporation parameter normalized to the value close to the solar neighborhood (E2000). Numerically, we find

$f_{\Sigma}=21.5\left(\frac{f_{\mathrm{c}}}{e^{-1}}\right)^{-1}\left(\frac{\gamma}{2.5}\right)\left(\frac{\phi_{k}}{0.01}\right)^{-1}\left(\frac{a_{\mathrm{l}}}{\mathrm{pc}}\right)^{2}$.

Following MO77 and E2000, we can also obtain the temperature of the hot gas

$T_{\mathrm{h}}=6.6 \times 10^{5}\left(S_{-13} \mathrm{E}_{51} f_{\Sigma} / \gamma\right)^{0.29} \mathrm{~K}$,

and the mass evaporation rate per unit volume

$\dot{\rho}_{\mathrm{ev}}=2.7 \times 10^{-10} S_{-13}^{0.71} \gamma^{0.29} \mathrm{E}_{51}{ }^{0.71} f_{\Sigma}^{-0.29} \mathrm{M}_{\odot} \mathrm{pc}^{-3} \mathrm{yr}^{-1}$, 
respectively, where $S_{-13}$ is defined as the the SN explosion rate in unit of $10^{-13} \mathrm{pc}^{-3} \mathrm{yr}^{-1}$. From equations (11), (12) and the definition of $f_{\Sigma}$ [eq. (9)], we see that the temperature $T_{\mathrm{h}}$ and the mass evaporation rate $\dot{\rho}_{\mathrm{ev}}$ are in fact independent of the parameter $\gamma$. Note that $\dot{\rho}_{\text {ev }}$ increases with supernova rate density (which is proportional to the SFR density) as a power of 0.71 . Thus, for a given total star formation, the total outflow rate increases with the volume of the star formation region.

The hot phase will first expand to form super bubbles which compress the ambient ISM. The compressed ambient ISM will be driven to form a "wind" with a velocity comparable to the isothermal sound speed of the hot phase, which is given by

$C_{i}=\left(k T_{\mathrm{h}} / \mu m_{\mathrm{p}}\right)^{1 / 2}=37 T_{5}^{1 / 2} \mathrm{~km} \mathrm{~s}^{-1}$

with $T_{5}$ the hot gas temperature in unit of $10^{5} \mathrm{~K}$ and $\mu$ the mean molecular weight per particle; we take $\mu=0.61$, a value appropriate for a fully ionized primordial gas. Because of the conservation of the specific enthalpy, the wind will accelerate and reach a bulk terminal speed to form a superwind (E2000). The terminal wind velocity is related to the isothermal sound speed by

$v_{\text {wind }}=\Gamma_{w} C_{i}$

We take $\Gamma_{w} \approx \sqrt{2.5}$, instead of a value $\sqrt{5}$; our value is appropriate when part of the thermal energy is lost radiatively [see Appendix B in E2000 for details]. Observationally, only when the initial sound speed of the hot gas is larger than $\sim 100 \mathrm{~km} \mathrm{~s}^{-1}$, can galactic winds be readily observed (e.g. Heckman et al 2000). From eq. (14), this sound speed corresponds to roughly $160 \mathrm{~km} \mathrm{~s}^{-1}$ in the terminal velocity.

Note that dark matter halos, which dominate the potential wells of galaxies, have not entered our discussions explicitly. Thus, the local properties of outflows, such as wind velocity and evaporation rate per unit volume, depend only on the properties of the ISM in the starforming region. This is consistent with the observations of X-rays from hot gas in galaxies and the detailed analysis of wind velocities by Heckman et al (2000), who found that terminal velocities of the superwinds are almost independent of the galaxy potential wells. However, the dark halo of a galaxy will play an important role in determining whether the outflow can eventually escape from the galaxy or fall back into the galaxy.

It is clear from equations (11) and (12) that the model predictions for the wind properties (velocity and mass outflow rate) depend on the choices of the values of $\phi_{k}$ and $a_{1}$. As discussed above, the values of these two parameters are probably in the ranges $0.01 \lesssim \phi_{k} \lesssim 0.1$ and 
$0.5 \mathrm{pc} \lesssim a_{1} \lesssim 1 \mathrm{pc}$. Using equations (11) and (12), one can estimate that these ranges correspond to a factor of $\lesssim 2$ in the predicted wind velocity, and a factor of $\lesssim 3$ in the predicted mass outflow rate. These factors should serve as an indication of the uncertainties in our model predictions.

One assumption we made is that the porosity $P$ retains close to unity. While this seems to be supported by observation but hard to prove rigorously (Silk 2001), it is important to examine the dependence of our results on the value of $P$. In general, galactic winds are more efficient during the early adiabatic stage of SN remnant evolution, when the porosity $P \lesssim 1$. In this case, SN remnants do not overlap with each other. The physical prescriptions we developed for $P=1$ are valid for $P<1$, except that the wind velocity $v_{\text {wind }}$ and

mass outflow rate $\dot{\rho}_{\mathrm{ev}}$ must be modified by a factor of $P^{-1 / 7}$ and $\left(1-e^{-P}\right) /\left[P^{5 / 7}\left(1-e^{-1}\right)\right]$, respectively (MO77). For a range of $P$ from 0.2 to 2 , the wind velocity changes by less than $25 \%$ while the mass outflow rate changes by less than $20 \%$ from our canonical results (for $P=1$ ). Clearly both $v_{\text {wind }}$ and $\dot{\rho}_{\text {ev }}$ are insensitive to $P$. As SN remnants evolve, they may overlap with each other and $P$ may becomes larger than 1 . In this case, the model will break down. As discussed above, in real galaxies, $P$ may be regulated so that its value cannot be much larger than 1 . We therefore believe that the assumption $P=1$ does not lead to significant error in our results.

\subsection{Star Formation in Spherical Regions}

So far we have only considered superwinds and mass outflows launched locally from a particular location in a star formation region. The derived superwinds and mass outflows therefore only depend on the local star formation activities. The observed wind velocity and mass outflow rate from a galaxy should be a proper average and sum of the wind velocity and outflow rate over the whole star formation region. It is these global quantities that can be directly compared with observation. In this subsection, we consider a simple model where star formation takes place uniformly in a spherical region. Here the wind and outflow are simply evaluated by the SFR density and the integration over the volume within star forming region. We treat individual star forming cells as source of winds but ignore the detailed transfer of energy and mass from the center to the outer region. Our treatment is obviously simplistic; a more careful consideration must consider wind interactions which result in the eventual outflow from the surface of the spherical region. 
For a spherical region of uniform star formation, $S_{-13}$ can be written as

$S_{-13}=\frac{\dot{M}_{*}}{M_{\mathrm{ps}} V}=2 \times 10^{3}\left(\frac{\dot{M}_{*}}{100 \mathrm{M}_{\odot} \mathrm{yr}^{-1}}\right)\left(\frac{M_{\mathrm{ps}}}{125 \mathrm{M}_{\odot}}\right)^{-1}\left(\frac{R}{1 \mathrm{kpc}}\right)^{-3}$,

where $\dot{M}_{*}$ is the SFR within radius $R$ and $M_{\mathrm{ps}}$ is the stellar mass of star formation per supernova explosion. For a Salpeter mass function $\left(n(M) d M \propto M^{-2.35} d M\right), M_{\mathrm{ps}} \approx 125 \mathrm{M}_{\odot}$ with a stellar mass range from 0.1 to $50 \mathrm{M}_{\odot}$.

Using equation (14), we can estimate the galactic wind terminal velocity

$v_{\text {wind }}=623 \mathrm{~km} \mathrm{~s}^{-1}\left[\left(\frac{\dot{M}_{*}}{100 \mathrm{M}_{\odot} \mathrm{yr}^{-1}}\right)^{0.29}\left(\frac{M_{\mathrm{ps}}}{125 \mathrm{M}_{\odot}}\right)^{-0.29}\left(\frac{R}{1 \mathrm{kpc}}\right)^{-0.87} \mathcal{K}^{0.29}\right]^{1 / 2}$

and the corresponding total mass evaporate rate

$\dot{M}_{\mathrm{ev}}=133 \mathrm{M}_{\odot} \mathrm{yr}^{-1}\left(\frac{\dot{M}_{*}}{100 \mathrm{M}_{\odot} \mathrm{yr}^{-1}}\right)^{0.71}\left(\frac{M_{\mathrm{ps}}}{125 \mathrm{M}_{\odot}}\right)^{-0.71}\left(\frac{R}{1 \mathrm{kpc}}\right)^{0.87} \mathcal{K}^{-0.29}$,

For convenience we have defined $\mathcal{K}$ as a function of $f_{\Sigma}$ and $\gamma$ as

$\mathcal{K}=\left(\frac{f_{\Sigma}}{21.5}\right)\left(\frac{\gamma}{2.5}\right)^{-1}$

which gives the dependence of $v_{\text {wind }}$ and $\dot{M}_{\mathrm{ev}}$ on the properties of the ISM.

For fixed $\mathcal{K}$, the wind velocity depends only on the SFR density, $\dot{M}_{*} / R^{3}$, because the temperature of the hot gas, hence the wind velocity of the driven ISM, depends only on the SN explosion rate density [cf. eq. (12)]. The wind velocity increases with the SFR density. Thus, galaxies with a more compact cold gas distribution will produce winds with higher velocity, no matter what the total amount of cold gas and total SFR are. This implies that superwinds are more likely to be observed in local starbursts and high-redshift star forming galaxies where the gas density is high and star formation activity is concentrated. For a given SFR density, the wind velocity increases with the lower limit of the cloud radii, because larger clouds leads to a larger value of $f_{\Sigma}$ [cf. eq.(9)]. The wind velocity also increases with decreasing $\phi_{k}$, because a higher $\phi_{k}$ implies a higher energy loss due to thermal transfer. As discussed in the last subsection, we adopt the lower limit of the wind velocity to be $\sim 160 \mathrm{~km} \mathrm{~s}^{-1}$ which, according to Heckman (2000), is the lower limit that can lead to an observable superwind. This limit directly translates into a lower limit on the SFR density, $0.01-0.1 \mathrm{M}_{\odot} \mathrm{yr}^{-1} \mathrm{kpc}^{-3}$, below which no observable wind is expected.

On the other hand, the mass outflow rate has an additional dependence on the size of star formation region. In Fig. 1, we show the predicted mass outflow rate as a function of SFR for three sets of parameters. The solid, dashed and dotted lines denote the results for $\left(\phi_{k}, a_{\mathrm{l}}\right)$ equal to $(0.1,0.5 \mathrm{pc}),(0.01,0.5 \mathrm{pc})$, and $(0.01,1 \mathrm{pc})$, respectively. The thin and 


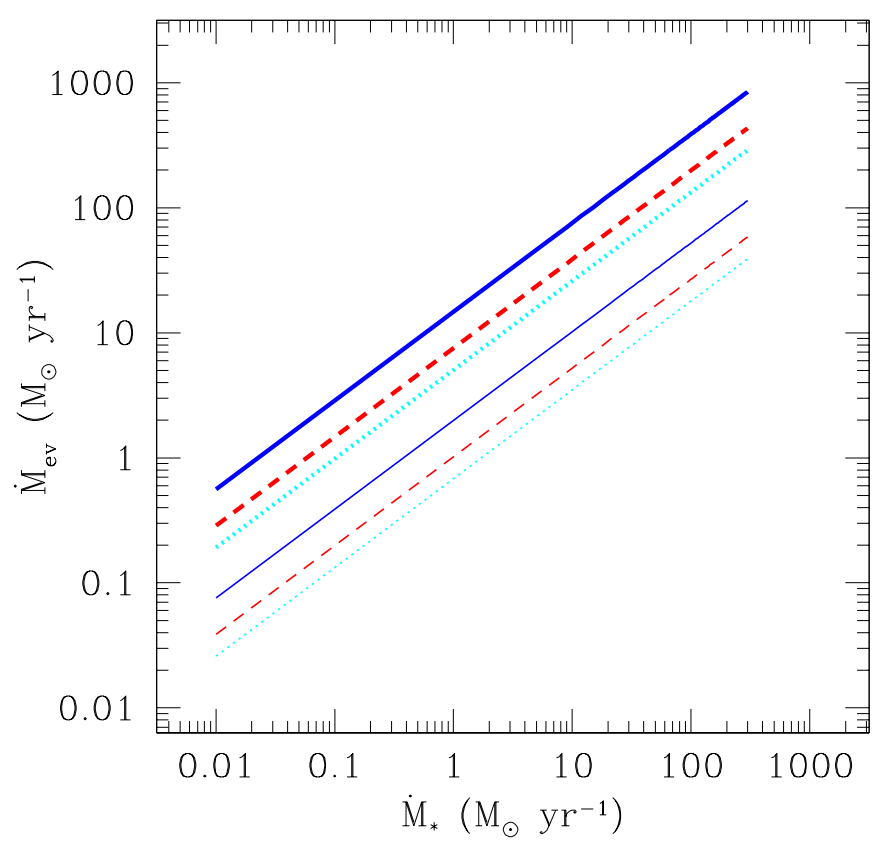

Figure 1. The predicted mass outflow rate as a function of the SFR for three sets of parameters, shown as the solid, dashed and dotted lines corresponding to $\left(\phi_{k}, a_{1}\right)$ equal to $(0.1,0.5 \mathrm{pc}),(0.01,0.5 \mathrm{pc})$, and $(0.01,1 \mathrm{pc})$, respectively. The thin and thick lines are for two different sizes of star formation regions, $0.1 \mathrm{kpc}$ and $1 \mathrm{kpc}$, respectively.

thick lines show results for the two adopted size of star formation region, $0.1 \mathrm{kpc}$ and $1 \mathrm{kpc}$, respectively. For a given total SFR, a larger star formation region gives a larger mass outflow rate, because the increase of the outflow rate density with SFR density is slower than linear (see eq. 12). Note, however, this increase of $\dot{M}_{\mathrm{ev}}$ with size cannot go indefinitely, because our assumption that $P=1$ will fail when the gas density becomes so low that the volume is too large for the SN remnants to fill.

Since the total power in the wind, which is proportional to $\dot{M}_{\mathrm{ev}} v_{\text {wind }}^{2}$, should be proportional to the total SFR, the dependence of $\dot{M}_{\mathrm{ev}}$ on model parameters can be understood in the same way as the dependence of wind velocity on model parameters. From Fig. 1 we see that the predicted mass outflow rates are comparable to (or higher than) the corresponding star formation rates for a wide range of model parameters. This is consistent with observation, e.g. Heckman et al. (2000) and Pettini et al. (2000; 2001; 2002).

\subsection{Star Formation in Galactic Disks}

In this subsection, we consider another case where star formation is assumed to be in thin galactic disks. This may be more realistic than the spherical model described above, because 
the cold gas supporting star formation generally has angular momentum and is found in a rotation-supported thin disk.

For simplicity, we assume the velocity dispersion $\left(\sigma_{g}\right)$ of the cold clouds in a disk to be constant everywhere. Throughout this paper, we choose $\sigma_{g}$ to be $10 \mathrm{~km} \mathrm{~s}^{-1}$, a value consistent with observation (Stark \& Brand 1989). The equation of hydrostatic equilibrium gives the following solution of cold gas density distribution in the vertical direction:

$\rho(z)=\frac{\mu_{\mathrm{g}}}{2 H_{g}} \operatorname{sech}^{2}\left(\frac{z}{H_{g}}\right)$

where $\mu_{\mathrm{g}}$ is the cold gas surface density and $H_{g}$ is the scale height given by

$H_{g}=\frac{\sigma_{g}^{2}}{\pi G \mu_{\mathrm{g}}}=74\left(\frac{\mu_{\mathrm{g}}}{\mathrm{M}_{\odot} \mathrm{pc}^{-2}}\right)^{-1}\left(\frac{\sigma_{g}}{\mathrm{~km} \mathrm{~s}^{-1}}\right)^{2} \mathrm{pc}$,

and $G$ is the gravitational constant (Spitzer 1942).

Kennicutt (1998) studied star formation in a wide range of physical conditions, ranging from quiescent galactic disks to starburst regions. He derived an empirical law for the SFR per unit area as a function of the cold gas surface density,

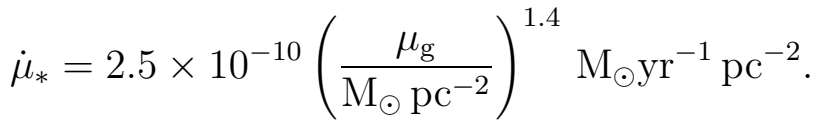

It then follows that the SFR density is

$\dot{\rho}_{*}=\frac{\dot{\mu}_{*}}{2 H_{g}}=1.7 \times 10^{-12}\left(\frac{\mu_{\mathrm{g}}}{\mathrm{M}_{\odot} \mathrm{pc}^{-2}}\right)^{2.4}\left(\frac{\sigma_{g}}{\mathrm{~km} \mathrm{~s}^{-1}}\right)^{-2} \mathrm{M}_{\odot} \mathrm{yr}^{-1} \mathrm{pc}^{-3}$,

and the SN explosion rate $S_{-13}$ is

$S_{-13}=10^{13} \times \frac{\dot{\rho}_{*}}{M_{\mathrm{ps}}}=1.35 \times 10^{-1}\left(\frac{\mu_{\mathrm{g}}}{\mathrm{M}_{\odot} \mathrm{pc}^{-2}}\right)^{2.4}\left(\frac{\sigma_{g}}{\mathrm{~km} \mathrm{~s}^{-1}}\right)^{-2}\left(\frac{M_{\mathrm{ps}}}{125 \mathrm{M}_{\odot}}\right)^{-1}$,

where $M_{\mathrm{ps}}$ is again the mass of stars formation corresponding to one supernova explosion.

Substituting the above equation into eqs. (11) and (14), we can infer the wind velocity $v_{\text {wind }}$ to be

$v_{\text {wind }}=150 \mathrm{~km} \mathrm{~s}^{-1}\left(\frac{\mu_{\mathrm{g}}}{\mathrm{M}_{\odot} \mathrm{pc}^{-2}}\right)^{0.35}\left(\frac{\sigma_{g}}{\mathrm{~km} \mathrm{~s}^{-1}}\right)^{-0.29}\left(\frac{M_{\mathrm{ps}}}{125 \mathrm{M}_{\odot}}\right)^{-0.15} \mathcal{K}^{0.15}$.

The corresponding mass outflow rate per unit area can be obtained by multiplying the mass outflow rate per unit volume and the scale height

$\dot{\mu}_{\mathrm{ev}} \approx 2 H_{g} \dot{\rho}_{\mathrm{ev}}=5.1 \times 10^{-9} \mathrm{M}_{\odot \mathrm{yr}^{-1}} \mathrm{pc}^{-2}\left(\frac{\mu_{\mathrm{g}}}{\mathrm{M}_{\odot} \mathrm{pc}^{-2}}\right)^{0.7}\left(\frac{\sigma_{g}}{\mathrm{~km} \mathrm{~s}^{-1}}\right)^{0.58}\left(\frac{M_{\mathrm{ps}}}{125 \mathrm{M}_{\odot}}\right)^{-0.71} \mathcal{K}^{-0.29}$,

where $\mathcal{K}$ is defined in eq. (18).

As one can see, for given $\sigma_{g}$ and $\mathcal{K}$, the wind velocity increases with $\mu_{\mathrm{g}}$. Thus, superwinds are more likely to occur in compact systems of cold gas. The wind velocity decreases with 


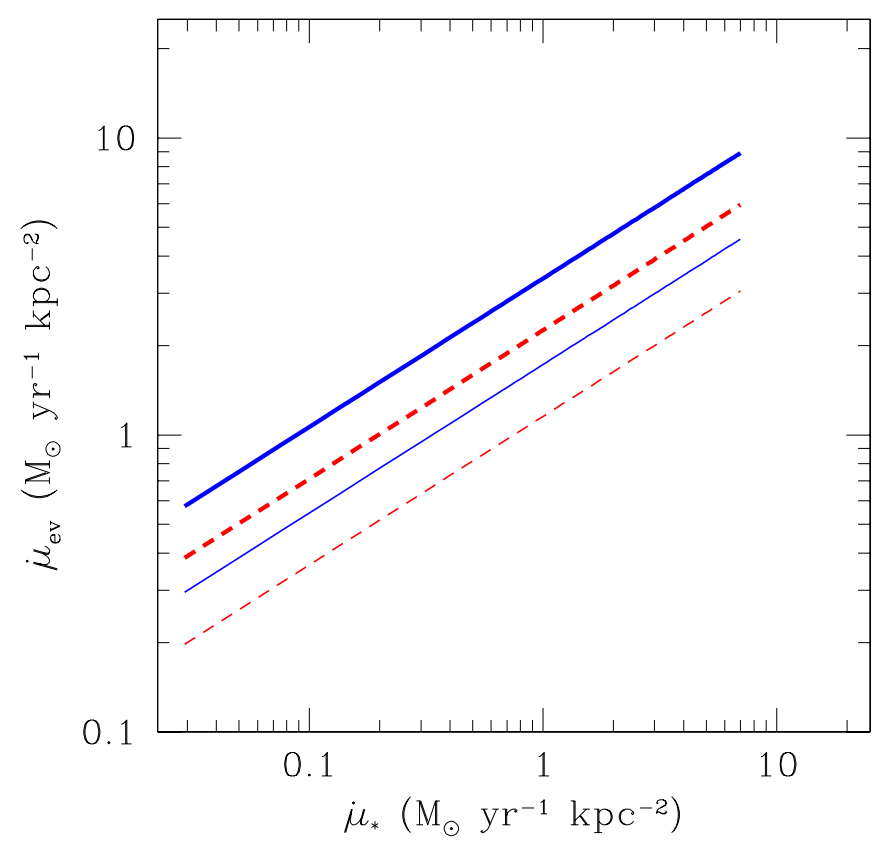

Figure 2. The predicted mass outflow rate per unit area as a function of the SFR per unit area. The solid and dashed lines are the results where the lower limit of the cloud radius is chosen to be $0.5 \mathrm{pc}$ and $1 \mathrm{pc}$, respectively. The thin and thick lines denote results with the parameter $\phi_{k}$ chosen to be 0.01 and 0.1 , respectively.

$\sigma_{g}$, because a higher value of $\sigma_{g}$ implies a larger disk thickness, and hence a lower density of cold gas. The mass outflow rate per unit area also increases with gas surface density, because of the dependence of the SFR per unit area on the surface density of cold gas.

As for the spherical star formation region in last subsection, we adopt the same threshold for the wind velocity, below which winds may not yield observable signatures (Heckman et al. 2000). This threshold corresponds to a lower limit on the SFR per unit area, which can be obtained from eq. (24). For the reasonable ranges of $\phi_{k}$ and $a_{1}$, i.e., $\phi_{k}=0.01-0.1$ and $a_{1}=0.5-1 \mathrm{pc}$, the limit is about

$\mathrm{SFR}_{\text {th }} \sim 0.01-0.05 \mathrm{M}_{\odot} \mathrm{yr}^{-1} \mathrm{kpc}^{-2}$

which, from eq. (21), corresponds to a cold gas surface density about $10-40 \mathrm{M}_{\odot} \mathrm{pc}^{-2}$. This limit is consistent with the observational estimation quoted by Heckman (2001).

The predicted mass outflow rate per unit area as a function of the star formation rate per unit area is shown in Fig. 2. The solid and dashed lines are results when the lower limit of the cloud radius is chosen to be $0.5 \mathrm{pc}$ and $1 \mathrm{pc}$, respectively. The thin and thick lines denote results with the parameter $\phi_{k}$ chosen to be 0.01 and 0.1 , respectively. For any given $\phi_{k}$ and $a_{1}$, the mass outflow rate per unit area increases with the SFR per unit area, as pointed out before. Note that the predicted mass outflow rates per unit area are comparable 
to (or higher than) the SFRs per unit area, which is consistent with observation (Heckman et al 2000; Pettini et al 2000; 2001; 2002).

So far we have considered only local properties of winds in galactic disks. To facilitate direct comparison between model predictions and observations, we must consider globallyaveraged quantities of the wind. As a simple example, we assume that the cold gas is distributed in an exponential disk

$\mu_{\mathrm{g}}=\mu_{0} \exp \left(-r / r_{d}\right), \mu_{0}=\frac{M_{\mathrm{g}}}{2 \pi r_{d}^{2}}$

where $\mu_{0}$ is the central surface density of cold gas, $M_{\mathrm{g}}$ is the total cold gas mass and $r_{d}$ is the scale length for the disk. Note that our results are not sensitive to the assumed cold gas surface density distribution because the cold gas surface density enters the wind velocity and mass outflow rate only with moderate power-law indices (0.35 and 0.7$)$ in eqs. (24) and (25), respectively.

As discussed above, there is a threshold in the SFR per unit area (eq. 26), i.e., the lower limit of the cold gas surface density, above which observable galactic winds can be produced. If the central surface density $\mu_{0}$ for a disk galaxy is lower than this, no observable galactic wind will occur. When $\mu_{0}$ is above the threshold, the threshold implies a critical radius $r_{\mathrm{cr}}$ for any given $\mathcal{K}$, beyond which no outflow will occur. The critical radius satisfies

$\mu_{\mathrm{cr}}=\mu_{0} \exp \left(-r_{\mathrm{cr}} / r_{d}\right)$

It is easy to calculate the total mass outflow rate within this critical radius

$$
\begin{aligned}
\dot{M}_{\mathrm{ev}}= & 6.2 \times 10^{-2} \mathrm{M}_{\odot \mathrm{yr}^{-1}}\left(\frac{r_{d}}{\mathrm{kpc}}\right)^{2}\left(\frac{\mu_{0}}{\mathrm{M}_{\odot} \mathrm{pc}^{-2}}\right)^{0.7} \\
& \times\left(\frac{\sigma_{g}}{\mathrm{~km} \mathrm{~s}^{-1}}\right)^{0.58}\left(\frac{M_{\mathrm{ps}}}{125 \mathrm{M}_{\odot}}\right)^{-0.71} \mathcal{K}^{-0.29} \mathcal{F}\left(0.7, \mu_{0}\right) \\
= & 9.8 \mathrm{M}_{\odot \mathrm{yr}^{-1}}\left(\frac{M_{\mathrm{g}}}{10^{9} \mathrm{M}_{\odot}}\right)\left(\frac{\mu_{0}}{\mathrm{M}_{\odot} \mathrm{pc}^{-2}}\right)^{-0.3} \\
& \times\left(\frac{\sigma_{g}}{\mathrm{~km} \mathrm{~s}^{-1}}\right)^{0.58}\left(\frac{M_{\mathrm{ps}}}{125 \mathrm{M}_{\odot}}\right)^{-0.71} \mathcal{K}^{-0.29} \mathcal{F}\left(0.7, \mu_{0}\right),
\end{aligned}
$$

where the function $\mathcal{F}\left(x, \mu_{0}\right)$ is defined as

$\mathcal{F}\left(x, \mu_{0}\right)=1-\left[1-x \ln \left(\frac{\mu_{\mathrm{cr}}}{\mu_{o}}\right)\right]\left(\frac{\mu_{\mathrm{cr}}}{\mu_{o}}\right)^{x}$.

$\mathcal{F}\left(x, \mu_{0}\right)$ describes the fraction of area that contributes to the observable galactic wind. For any given $x, \mathcal{F}$ will first increase with $\mu_{0}$ for small values of $\mu_{0}$, and then decrease with increasing $\mu_{0}$ for large values of $\mu_{0}$. 


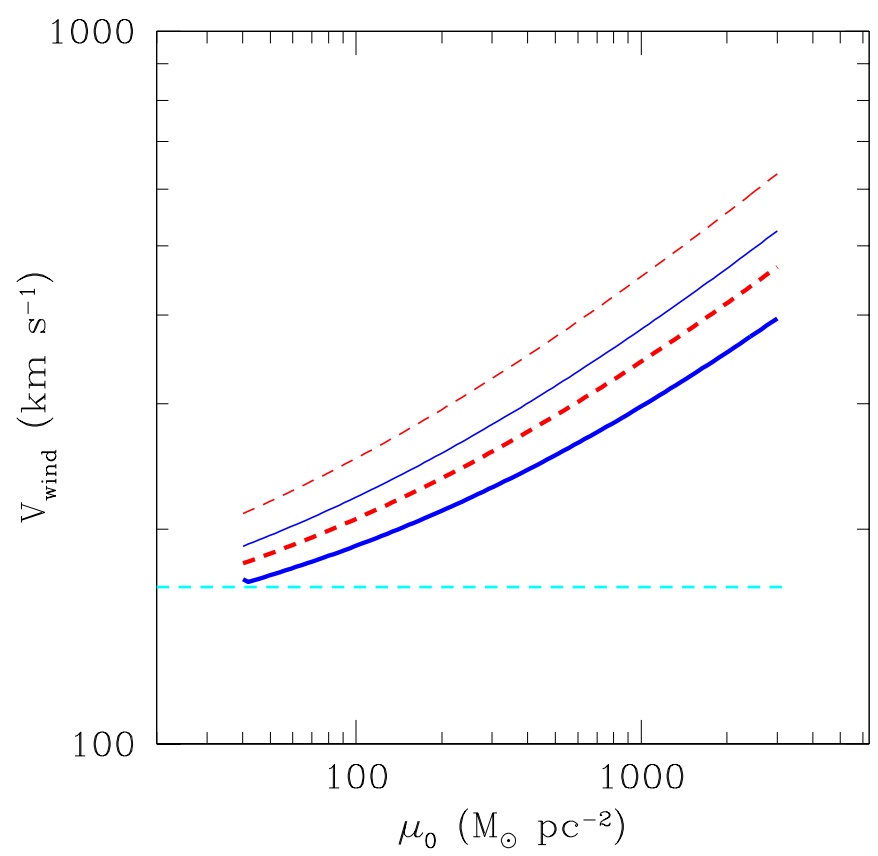

Figure 3. The predicted mass outflow rate weighted wind velocity as a function of $\mu_{0}$ where the solid and dashed lines have the same notations as that in Fig. 2 with the dotted horizon line marking the lower limit of the velocity, $\sim 160 \mathrm{~km} \mathrm{~s}{ }^{-1}$. Here the velocity dispersion of the cold gas clouds is assumed to be $10 \mathrm{~km} \mathrm{~s}^{-1}$.

The observed superwind velocity is an appropriate average of the wind velocities over the whole disk. Since the mass outflow rate per unit area is proportional to $\mu_{\mathrm{g}}^{0.7}$, we define the global wind velocity to be the average of the wind velocities weighted by $\mu_{\mathrm{g}}^{0.7}$. This wind velocity is

$V_{\text {wind }}=68 \mathrm{~km} \mathrm{~s}^{-1}\left(\frac{\mu_{0}}{\mathrm{M}_{\odot} \mathrm{pc}^{-2}}\right)^{0.35}\left(\frac{\sigma_{g}}{\mathrm{~km} \mathrm{~s}^{-1}}\right)^{-0.29}\left(\frac{M_{\mathrm{ps}}}{125 \mathrm{M}_{\odot}}\right)^{-0.15}\left(\frac{\mathcal{F}\left(1.05, \mu_{0}\right)}{\mathcal{F}\left(0.7, \mu_{0}\right)}\right) \mathcal{K}^{0.15}$.

In reality, the observed wind velocity depends on where the wind originates, and our weighting scheme can only serve as an approximation. We have also made calculations using a weight proportional to $\mu_{\mathrm{g}}$ and to $\mu_{\mathrm{g}}^{1.4}$ (i.e. to the surface density of SFR). The results for these three weighting schemes differ only by $20 \%$. Note that for given $\mathcal{K}$ the wind velocity for an exponential disk depends only on the central surface density of cold gas, while the total mass outflow rate for the disk depends in addition on the scale length (or the total cold gas mass).

Fig. 3 shows the the predicted global wind velocity for an exponential disk with $\mu_{0} \gtrsim \mu_{\mathrm{cr}}$ as a function of cold gas central surface density $\mu_{0}$ for four different combinations of $\phi_{k}$ and $a_{\mathrm{l}}$. The notation of the figure is the same as that in Fig. 2 with the dotted horizon line marking the lower limit of the velocity, $\sim 160 \mathrm{~km} \mathrm{~s}^{-1}$, as discussed above. For given $\phi_{k}$ 


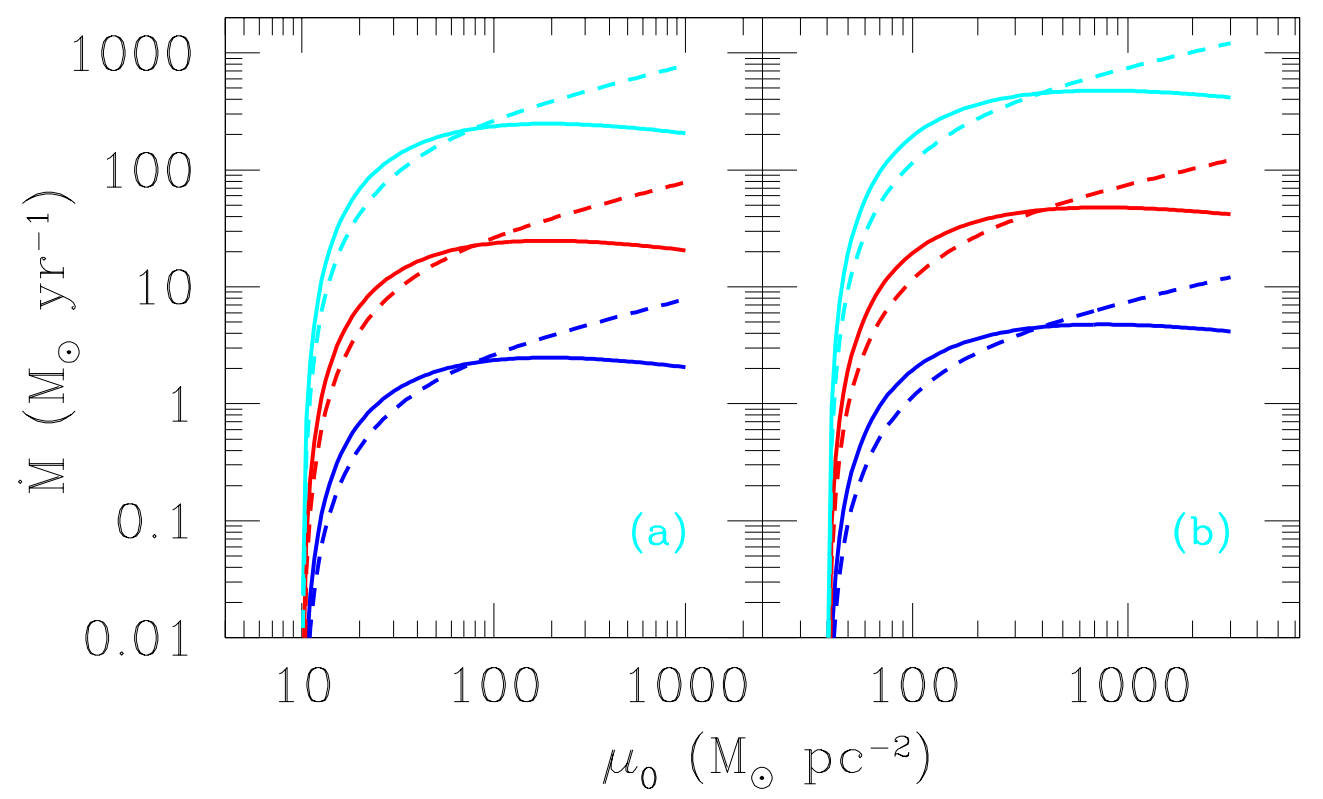

Figure 4. The predicted mass outflow rate (solid lines) and the corresponding star formation rate (dashed lines) as a function of $\mu_{0}$ for given the total cold gas mass, where lines from top to bottom denote the total cold gas mass to be $2 \times 10^{11} \mathrm{M}_{\odot}$, $2 \times 10^{10} \mathrm{M}_{\odot}$ and $2 \times 10^{9} \mathrm{M}_{\odot}$ respectively. Here the velocity dispersion of the cold gas clouds is assumed to be $10 \mathrm{~km} \mathrm{~s}^{-1}$, and (a) and (b) denote the results of $\left(\phi_{k}, a_{1}\right)$ equal to $(0.01,1 \mathrm{pc})$ and $(0.1,0.5 \mathrm{pc})$, respectively.

and $a_{1}$, the predicted global wind velocities increase with the cold gas surface density $\mu_{0}$. Galaxies with low cold gas surface density (e.g., low surface brightness galaxies or highsurface brightness galaxies with low cold gas surface density such as our Milky Way) will not produce observable galactic winds because their star formation rate is too low.

As an example, Fig. 4 plots the predicted total mass outflow rate (solid lines) and the corresponding SFR (dashed lines) as a function of $\mu_{0}$ with (a) and (b) denoting the results of $\left(\phi_{k}, a_{\mathrm{l}}\right)$ equal to $(0.01,1 \mathrm{pc})$ and $(0.1,0.5 \mathrm{pc})$, respectively. The lines from top to bottom are results for a total cold gas mass to be $2 \times 10^{11} \mathrm{M}_{\odot}, 2 \times 10^{10} \mathrm{M}_{\odot}$ and $2 \times 10^{9} \mathrm{M}_{\odot}$, respectively. From the figure we see that the cold gas central density $\mu_{0}$ must be larger than a critical value $\mu_{\mathrm{cr}}$ (about 10 and $40 \mathrm{M}_{\odot} \mathrm{pc}^{-2}$ for the values of $\phi_{k}$ and $a_{1}$ adopted here respectively) for an observable wind to occur. If $\mu_{0}<\mu_{\mathrm{cr}}$, no observable galactic wind will be produced no matter how large the total cold gas mass is.

For $\mu_{0} \gtrsim \mu_{\mathrm{cr}}$, the total mass outflow rates increase with the total mass of cold gas for a given $\mu_{0}$ because of the increase of the SFR and the star formation region which can produce observable outflows. For a given total cold gas mass, the predicted mass outflow rate first increases rapidly with $\mu_{0}$, because the gas mass that can produce observable winds [i.e. $r_{\mathrm{cr}}$ in eq. (28)] increases with $\mu_{0}$. The mass outflow rate as a function of $\mu_{0}$ reaches a plateau 
and then decreases when $\mu_{0}$ is larger than $\sim 10^{3} \mathrm{M}_{\odot} \mathrm{pc}^{-2}$. This happens because when $\mu_{0}$ is high enough, the total gas mass that can produce observable galactic winds is saturated while the SFR density increases with increasing $\mu_{0}$. This increase in SFR density reduces the mass outflow rate, again because the increase of $\dot{\rho}_{\mathrm{ev}}$ with SFR density is slower than linear (see eq. 12). Note that the predicted mass outflow rates are comparable to the SFR for a very wide range of $\mu_{0}$, which is consistent with current observations.

\section{COMPARISONS WITH OBSERVATIONS}

As discussed in the introduction, many observational studies have investigated galactic winds both in local starburst galaxies and in high-redshift star forming galaxies. In this section, we examine whether the predictions of our model can match the current observational data.

For definiteness, we will adopt $\phi_{k}=0.01$ and $a_{1}=1 \mathrm{pc}$ throughout this section. As we discussed in Sec. 2.1, other plausible choices of these parameters may result in a lower wind velocity by a factor of $\lesssim 2$ and a higher mass outflow rate by a factor of $\lesssim 3$. The choice is quite arbitrary, since the exact values of $\phi_{k}$ and $a_{1}$ appropriate for star forming galaxies are not known a priori. Our chosen values do give reasonable agreement with the observational results to be discussed below.

\subsection{Local starburst galaxies}

Based on the Na D absorption lines, Heckman et al (2000) estimated galactic wind velocities for local starburst galaxies. They found that the wind velocities are in the range of 400 to $800 \mathrm{~km} \mathrm{~s}^{-1}$. They also estimated mass outflow rates, which they claimed to be comparable to the SFRs. The observational results for the mass outflow rates are only qualitative and we have shown that they are consistent with our model predictions. In what follows, we will primarily focus on the wind velocities which are more accurately determined.

At first we assume that the star formation takes place within spherical regions. We can then estimate the global galactic winds for these galaxies using equation (16), provided that their SFRs and sizes are known. We estimate the SFRs for these starburst galaxies using their FIR luminosities (Kennicutt 1998)

$\frac{\mathrm{SFR}}{\mathrm{M}_{\odot} \mathrm{yr}^{-1}}=\frac{L_{\mathrm{FIR}}}{5.8 \times 10^{9} L_{\odot}}$.

The sizes of the star forming regions for the galaxies in consideration are not available. As a rough estimate, we take the sizes to be the same as the observational slit widths, which, (C) 2002 RAS, MNRAS 000, 000-000 


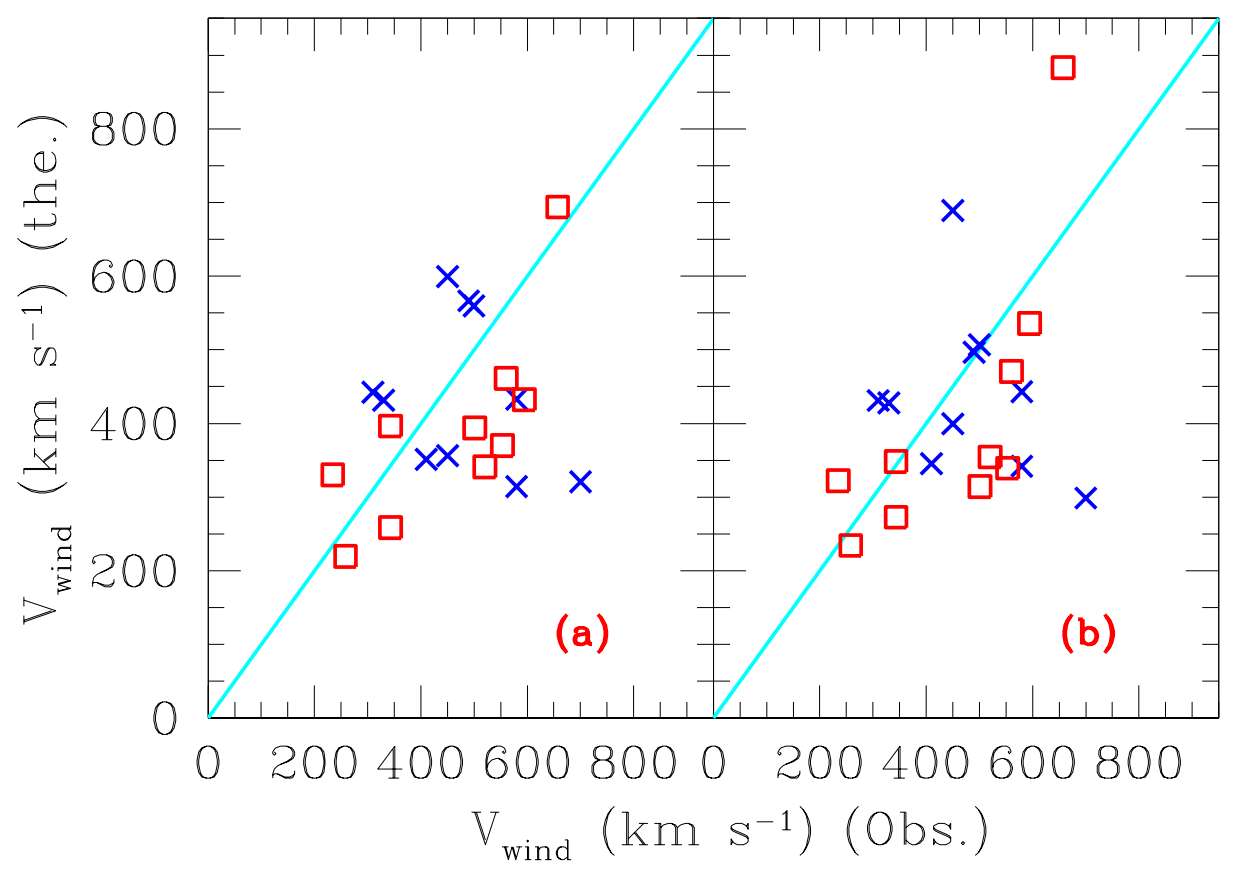

Figure 5. The predicted wind velocities vs observed values for local starburst galaxies. The crosses denote the observations of $\mathrm{Na} \mathrm{D}$ absorption lines and open squares denote the estimated results based on the the hot gas temperatures from ROSAT and ASCA. The lines denote the agreement between observation and model predictions. (a) for spherical star formation region; (b) for exponential star formation disk (see the text for details).

according to Heckman et al (2000), is a reasonable match to the typical sizes of powerful starbursts. The observational data are from Table 4 in Heckman et al (2000). The predicted wind velocities vs the observed ones are plotted as crosses in Fig. 5(a). The lines in the figure denote the agreement between observation and model predictions. As can be seen, our model predictions roughly match the observations within the model and observational uncertainties.

Another way to empirically estimate the super wind velocities for galaxies is based on X-ray observations. Galactic winds will spread out the hot gas within galactic halos and contribute to the soft component of extended X-ray emission. As the hot gas temperature can be inferred from X-ray observations (e.g., from ROSAT or ASCA), we can estimate the wind velocities from the temperature of the soft X-ray components using equation (11). The galaxies we selected are similar to those in Heckman et al (2000). They are NGC4449 (Della Ceca, Griffiths \& Heckman 1997), NGC2146 (Della ceca et al. 1999), NGC253, NGC3079, M82, NGC4631 (Dahlem, Weaver \& Heckman 1998), NGC1569 (Della ceca et al. 1996), Arp299 (Heckman et al 1999), NGC6240 (Iwasawa \& Comastri 1998), NGC1808 (Awaki et al. 1996). Note that we assume the relation between wind velocity and soft X-ray temperature 
to be $V_{\text {wind }}=\sqrt{2.5 k T_{X}}$ rather than $\sqrt{5 k T_{X}}$, the relation adopted by Heckman et al (2000). The reason for our choice is discussed below eq. (14).

We can estimate the SFRs of these galaxies based on their FIR luminosities according to equation (32). The sizes of their star formation regions are adopted from the corresponding $\mathrm{H} \alpha$ observations, which can be found in the references listed above. The predicted wind velocities can then be obtained using eq. (16). The open squares in Fig. 5(a) indicate the predicted wind velocities and the estimated values from the X-ray observations. We find that the predictions match the estimated values reasonably well.

As pointed out in Sec. 2.3, the predicted results are not sensitive to the assumed gas surface distribution if we assume starbursts take place in exponential disks. Assuming that the cold gas in these galaxies is distributed exponentially and that observed star formation region contain just one half of the total star formation activity in the galaxies, we can also predict the superwind velocities for the above starburst galaxies based on equation (31). The model predictions versus observational results are shown in Fig. 5(b). Here again, the model predictions match observation.

\subsection{Lyman break galaxies}

The UV dropout method has been very successful in identifying active star forming galaxies (Lyman break galaxies, hereafter LBGs) at a redshift of $z \approx 3$ (Steidel, Pettini \& Hamilton 1995). Much observational and theoretical work has investigated their physical nature (see Mo \& Fukugita 1996; Mo, Mao \& White 1999; Kauffmann et al. 1999; Katz, Hernquist \& Weinberg 1999; Shu 2000; Shu, Mao \& Mo 2001). Based on observations of Lyman alpha and nebular emission lines, LBGs are also inferred to display galactic winds (Pettini et al 2001; 2002; Adelberger et al 2002). LBGs display a wide range of wind velocities, 250 to nearly $1000 \mathrm{~km} \mathrm{~s}^{-1}$ with a median value about $500-600 \mathrm{~km} \mathrm{~s}^{-1}$. As for local starburst galaxies, the mass outflow rates for LBGs are difficult to establish observationally. Pettini et al (2000; 2002) measured the mass outflow rate for a specific LBG, the lensed and magnified MS 1512cB58; the value is $\sim 60 \mathrm{M}_{\odot} \mathrm{yr}^{-1}$, comparable to its SFR obtained from its UV luminosity.

At present it is unclear whether the star formation in LBGs occurs in quasi-spherical regions or more in a disk-like geometry. For this reason, we will consider both scenarios below in turn.

If the star formation activities in LBGs are quasi-spherical and homogeneous, then we 


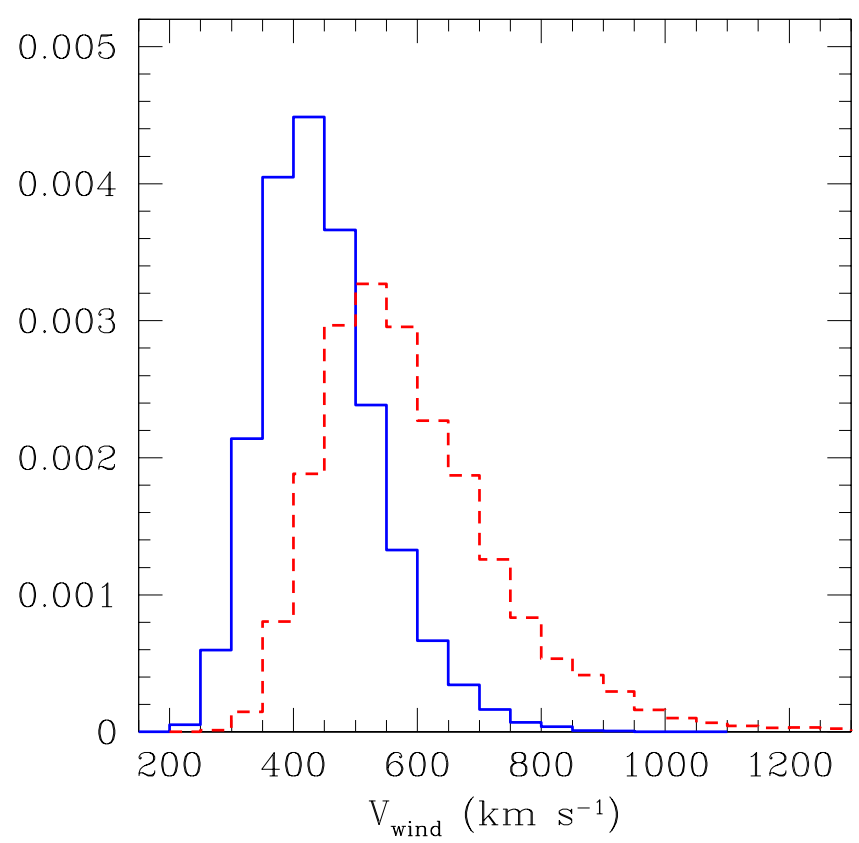

Figure 6. The predicted distribution of the galactic winds for LBGs. The solid and dashed histograms denote results where star formation is assumed to take place in spherical regions and thin exponential disks, respectively.

can use equations equations (16) and (17) to estimate the mass outflow rates and galactic wind velocities for LBGs if we know the star formation rates and the sizes of their star formation regions. Following the procedure outlined in Shu, Mao \& Mao (2001), we adopt the SFR distribution of LBGs from their dust-corrected UV luminosity and a log-normal size distribution. We use Monte Carlo simulations to select galaxies following these SFR and size distributions. We then substitute these two quantities into eqs. (16-17) to obtain the predicted distributions of the galactic wind velocities and the corresponding mass outflow rates. The results are shown as the solid histograms in Figures 6 and 7, respectively. The predicted winds have velocities in the range from 200 to $900 \mathrm{~km} \mathrm{~s}^{-1}$ and a median value of $450 \mathrm{~km} \mathrm{~s}^{-1}$, in agreement with the quite limited observational results. The predicted median value of the mass outflow rate is about $80 \mathrm{M}_{\odot} \mathrm{yr}^{-1}$, similar to the inferred value for MS $1512-c B 58$.

The second scenario we consider for LBGs is that their star formation occurs in a preassembled disk. For simplicity, we assume that the cold gas surface density has an exponential distribution. In this case, the model discussed in Sec. 2.3 can be applied to the LBG population. We use a procedure identical to that used in the spherical model to simulate the LBG population.

Based on eqs. (29-31), the estimated outflow rates and wind velocities can be obtained. 


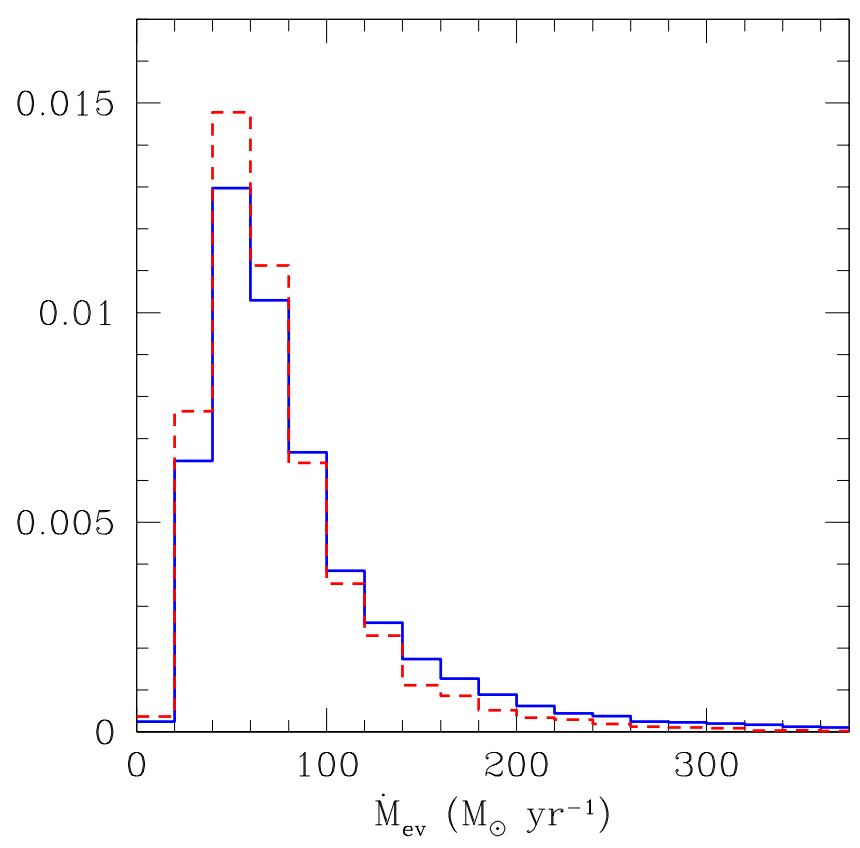

Figure 7. The predicted distribution of the mass outflow rates for LBGs. The solid and dashed histograms denote results where star formation is assumed to occur in spherical regions and thin exponential disks, respectively.

The dashed histograms in Figs. 6 and 7 show the model predictions for the distributions of the galactic wind velocities and the corresponding mass outflow rates for LBGs, respectively. It can be seen that the median value of the predicted wind velocities for LBGs is $\sim 600 \mathrm{~km} \mathrm{~s}^{-1}$. The median value of the predicted mass outflow rates is about $60 \mathrm{M}_{\odot} \mathrm{yr}^{-1}$. Both are consistent with observations.

\section{ESCAPING GALACTIC WINDS AS A FUNCTION OF $V_{\mathrm{C}}$ AND REDSHIFT}

As we discussed in Sec. 2, galaxies with more compact cold gas distributions (and hence more compact star formation activity) will produce stronger galactic winds. Galactic winds will thus occur preferentially in local starburst galaxies and high redshift galaxies. If the speed of a galactic wind is smaller than the escape velocity of the host galaxy, the mass outflow will fall back into the galaxy and form a galactic fountain. Otherwise, the outflow will escape from the host. This we call an "escaping" outflow. The mechanical energy of the outflow can heat the the intergalactic medium (IGM) while heavy elements contained in the wind can chemically enrich this gas. It is therefore important and interesting to estimate the fraction of galaxies (by number) that will have escaping outflows at different redshifts. We 


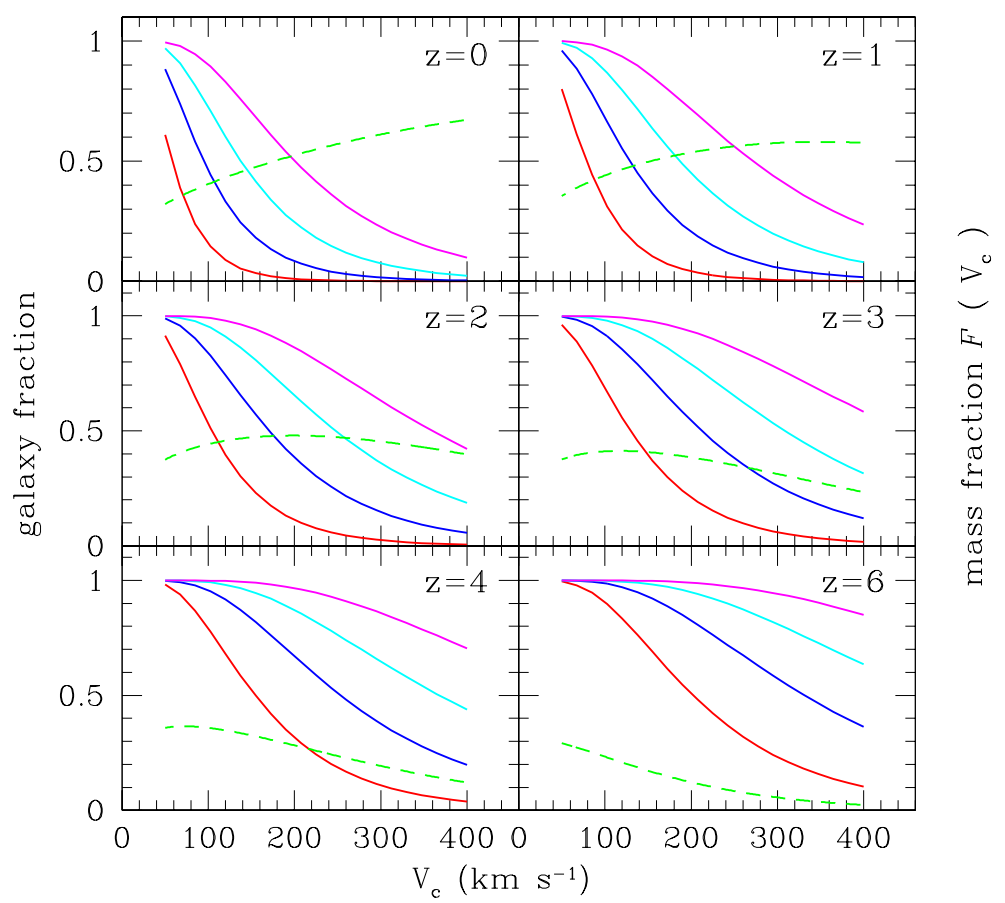

Figure 8. The predicted fraction of galaxies where the superwinds escape from their dark matter haloes is shown as a function of circular velocity at six different redshifts. In each panel, the four solid lines correspond to a gas faction of $m_{\mathrm{g}}=0.1,0.05$, 0.025 and 0.01 (from top to bottom), respectively. The predicted mass fraction $F\left(V_{\mathrm{c}}\right)$ in halos for a given $V_{\mathrm{c}}$ is shown as a dashed line at each redshift in the current $\Lambda \mathrm{CDM}$ cosmogony.

address this question in the currently preferred $\Lambda$ CDM cosmogony with a matter density $\Omega_{m}=0.3$, a cosmological constant $\Omega_{\lambda}=0.7$, a Hubble constant $h=70 \mathrm{~km} \mathrm{~s}^{-1} \mathrm{Mpc}^{-1}$; the power-spectrum is described by a shape parameter $\Gamma=0.2$ and the usual normalisation constant $\sigma_{8}=0.9$.

We assume that the distribution of cold gas within a galaxy is exponential; our results will not change significantly if we adopt other profiles (see Sec. 2.3). We use Mo, Mao \& White (1998) to model the formation of disks in dark matter haloes. In this model, the disk properties are determined by the halo circular velocity, $V_{\mathrm{c}}$, a dimensionless spin parameter, $\lambda$, and the fraction of total mass $\left(m_{\mathrm{g}}\right)$ that settles into the exponential disk. The spin parameter follows a log-normal distribution with a median $\bar{\lambda}=0.05$ and a dispersion $\sigma_{\ln \lambda}=0.5$ (e.g., Warren et al 1992; Lemson \& Kauffmann 1999). Once these three parameters $\left(V_{\mathrm{c}}, \lambda\right.$, and $m_{\mathrm{g}}$ ) are specified, the disk properties and hence the star formation and outflow properties can be evaluated using the formalism developed here. In Fig. 8, we show the number fraction of galaxies with escaping winds occuring in the disks as a function of circular velocity at six different redshifts. For each redshift, four solid lines are plotted, corresponding to four different $m_{\mathrm{g}}$ values. Note that, for these results, we have averaged over the spin parameter 
distributions. In the same figure, we also show the mass fraction $F\left(V_{\mathrm{c}}\right)$ in halos with a given circular velocity $V_{\mathrm{c}}$ in logrithmic bin at different redshifts, i.e.,

$F\left(V_{\mathrm{c}}\right) d \log \mathrm{V}_{\mathrm{c}}=\frac{d F\left(>V_{\mathrm{c}}, z\right)}{d \log \mathrm{V}_{\mathrm{c}}} d \log \mathrm{V}_{\mathrm{c}}$,

where $F\left(>V_{\mathrm{c}}, z\right)$ is the mass fraction in haloes with circular velocity larger than $V_{\mathrm{c}}$ as predicted by the updated Press-Schechter formalism (c.f. Mo \& White 2002). From the dashed line, one can easily estimate the mass fraction of total halos and the typical halo circular velocity which will produce escaping mass outflows.

As expected, for any given $m_{\mathrm{g}}$, a larger fraction of small halos produces escaping outflows at any redshift because of their shallower potential wells. Hence, during their evolution, a significant fraction of their baryons will be lost due to the galactic winds. It also implies that most small halos in the local universe are dark matter dominated which is consistent with observation (e.g. Persic, Salucci \& Stel 1996). Given $V_{\mathrm{c}}$, galaxies with larger $m_{\mathrm{g}}$ have a larger probability to produce an escaping outflow at any redshift because of their more active star formation. As the redshift increases, more and more galaxies produce escaping outflows because of the increase in their SFR densities. For example at $z=3$, more than $80 \%$ galaxies with $V_{\mathrm{c}} \lesssim 200 \mathrm{~km} \mathrm{~s}^{-1}$ will produce mass outflows that escape for $m_{\mathrm{g}} \gtrsim 0.05$. Hence escaping outflows will occur commonly in LBGs because the median value of their circular velocity is about $150 \mathrm{~km} \mathrm{~s}^{-1}$ and their $m_{\mathrm{g}}$ may be $\gtrsim 0.06$ (Shu, Mao \& Mo 2001). Hence, a significant number of galaxies will contribute to the heating of the IGM or ICM. Subsequent galaxy formation therefore will occur in preheated intergalactic media, as argued by Mo \& Mao (2001). Galactic winds therefore should be taken into account for high-redshift galaxies.

As a comparison, it is interesting to study how the star formation rate is partitioned in haloes with different circular velocities. Specifically, we study the differential probability distribution of star formation rate in logrithmic scales of $V_{c}$, i.e.,

$f\left(V_{\mathrm{c}}\right) d \log \mathrm{V}_{\mathrm{c}}=\frac{\mathrm{SFR}\left(\mathrm{V}_{\mathrm{c}}\right)}{\int \mathrm{SFR}\left(\mathrm{V}_{\mathrm{c}}\right) \mathrm{d} \log \mathrm{V}_{\mathrm{c}}} d \log \mathrm{V}_{\mathrm{c}}$,

where SFR is the star formation rate in galaxies for a given circular velocity $V_{\mathrm{c}}$ averaged over all spin parameter distributions. Note that this distribution function is normalised to unity for $V_{c}$ between $30 \mathrm{~km} / \mathrm{s}$ and $400 \mathrm{~km} / \mathrm{s}$.

We define the accumulative probability distribution function for galaxies with escaping outflows, $f_{\text {ro }}\left(<V_{\mathrm{c}}\right)$, as a function of $V_{\mathrm{c}}$,

$f_{\mathrm{ro}}\left(<V_{\mathrm{c}}\right)=\frac{\int_{30}^{V_{\mathrm{c}}} \mathrm{SFR}_{\mathrm{esc}}\left(\mathrm{V}_{\mathrm{c}}\right) \mathrm{dV}_{\mathrm{c}}}{\int_{30}^{400} \mathrm{SFR}\left(\mathrm{V}_{\mathrm{c}}\right) \mathrm{dV} \mathrm{V}_{\mathrm{c}}}$, 


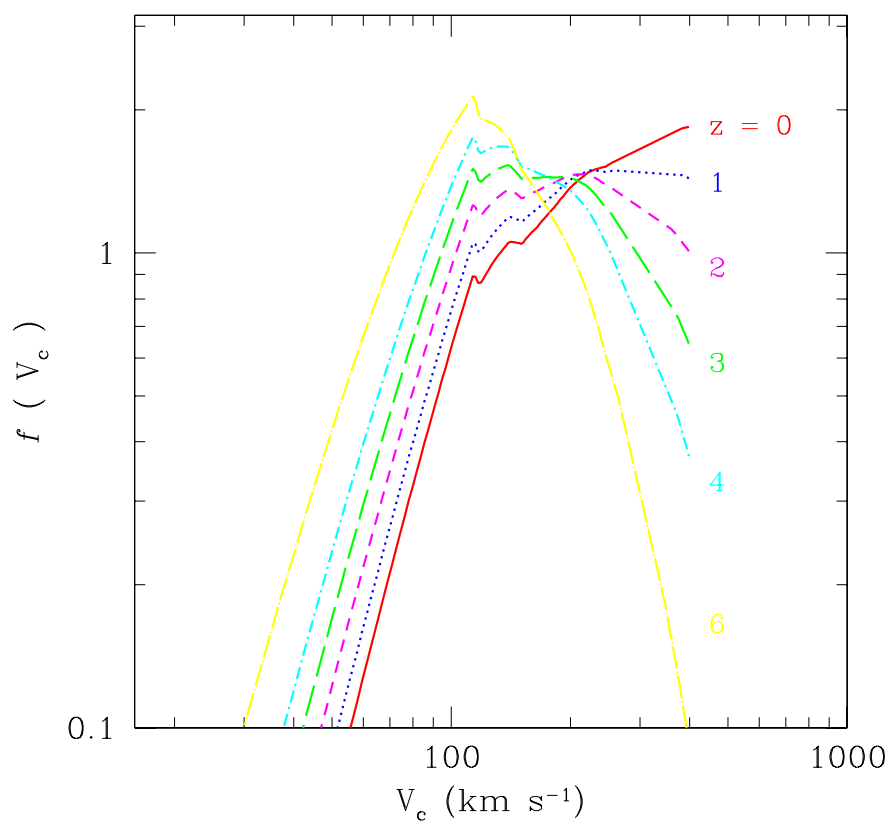

Figure 9. The predicted fraction of galaxies weighted by the SFR as a function of circular velocity at six different redshifts. Gas cooling and feedback effect have been taken into account and the currently favoured $\Lambda$ CDM cosmogony is adopted (see text for details).

where $\mathrm{SFR}_{\mathrm{esc}}$ is the star formation rate in galaxies with escaping outflows for a given circular velocity $V_{\mathrm{c}}$.

To obtain these two probability distributions, we have assumed that all galaxies are disk galaxies. Furthermore, the gas fraction $m_{\mathrm{g}}$ in the disks is taken to be the fraction of gas that eventually cool in their host haloes; we take the cooling function from Sutherland \& Dopita (1993) assuming the metallicity of 0.01 solar value. The influence of the feedback on $m_{\mathrm{g}}$ is also taken into account by adopting

$m_{\mathrm{g}}=\frac{m_{\mathrm{g} 0}}{1+\left(\frac{150 \mathrm{kms}^{-1}}{V_{\mathrm{c}}}\right)^{2}}$

as suggested by Dekel \& Silk (1986) and White \& Frenk (1991). Here $m_{\mathrm{g} 0}$ is the maximum baryon fraction that can cool in the haloes to form disks.

We show the two probability distributions in Figures 9 and 10 for six different redshifts, respectively. We can see from Fig. 9 that the SFR fraction of galaxies peaks at $V_{\mathrm{c}} \sim 100-200 \mathrm{kms}^{-1}$ at redshift $z>1$. However, at lower redshift, most of the SFR is contributed by more massive galaxies because the feedback at low redshift is not as efficient and most baryons within massive halos can cool to form stars. From Fig. 10, we see that the contribution of SFR for galaxies with escaping outflows are dominated by galaxies with 


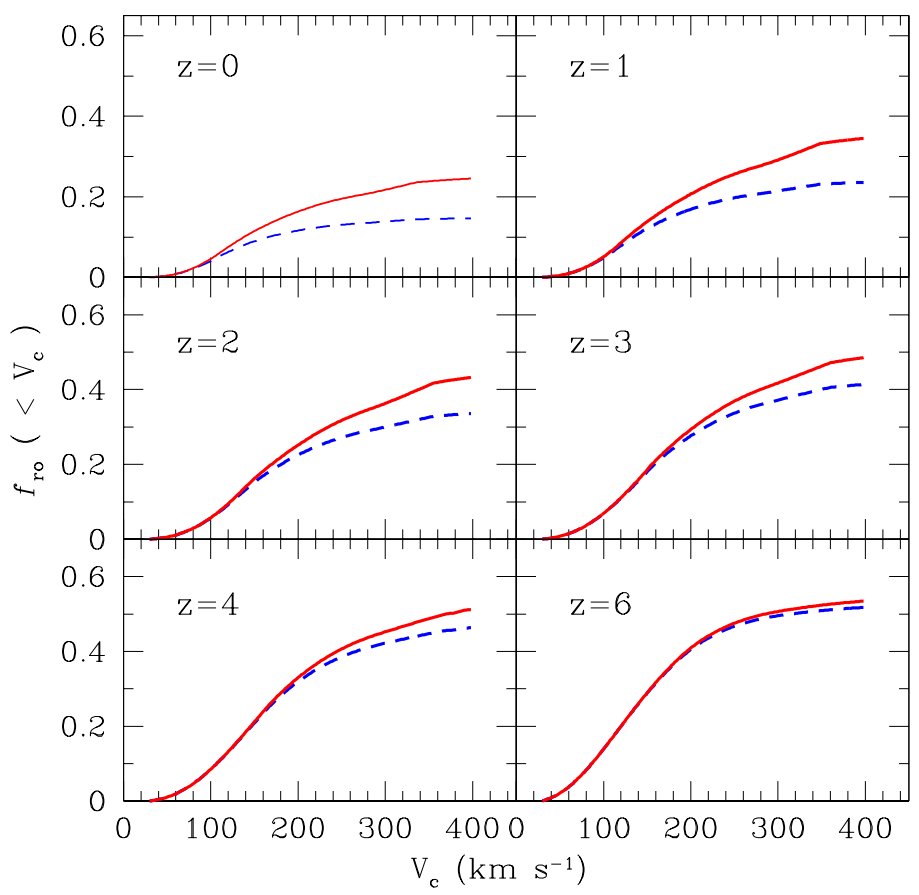

Figure 10. The predicted accumulative probability distribution function of galaxies with escaping outflows as a function of halo circular velocity at six different redshifts. For each redshift, the solid and dashed lines denote results for $m_{\mathrm{g} 0}=0.1$ and 0.05 , respectively. Gas cooling and feedback effect have been taken into account and the currently favoured $\Lambda$ CDM cosmogony is adopted (see text for details).

$V_{\mathrm{c}} \sim 200 \mathrm{~km} \mathrm{~s}^{-1}$ at redshift $z \lesssim 3$, while at higher $z$ significant contribution comes from systems with $V_{\mathrm{c}} \sim 150 \mathrm{~km} \mathrm{~s}^{-1}$. This is because, although at lower redshift the SFR is shifted toward systems with higher $V_{\mathrm{c}}$, escaping superwinds are more difficult to produce in these systems. At $z>3$, more than $40 \%$ of all stars are formed in systems where escaping winds are expected. Note that a change in the value of $m_{\mathrm{g} 0}$ does not affect the result significantly. At high redshift, the number density of high $V_{\mathrm{c}}$ systems goes down rapidly and so not many stars can form in these systems.

\section{SUMMARY}

In this paper, we develop a simple analytic model to understand superwinds and mass outflows that are observed in local starburst galaxies and high-redshift star-forming galaxies. Our scenario is based on the model of Mckee \& Ostriker (1977) for the evolution of SN remnants in the ISM, which is similar to Efstathiou (2000). We show that the properties of superwinds, such as wind velocity and mass outflow rate, depend not only on the properties of the ISM, but also on the global properties of star forming galaxies. Our main conclusions are: 
- Observable winds are produced only in systems where the density of SFR is higher than some threshold. This is consistent with the observational results given by Heckman (2001) and Heckman et al (2000), and implies that low surface-brightness galaxies and high surfacebrightness galaxies with low cold gas surface density cannot produce large-scale superwinds.

- The velocity of superwinds driven by SN explosions depends only on the SFR density. Galaxies in which current star formation is confined in a more compact region can produce winds with higher velocity. This implies that superwinds are expected in local starbursts and in high-redshift star-forming galaxies. The mass outflow rate depends, in addition, on the size of star formation region; a larger star formation region allows more mass to be loaded in the wind.

- The predicted mass outflow rates are comparable to or higher than the corresponding SFRs, consistent with current observations both for local starburst galaxies (Heckman et al 2000) and for high redshift star-forming galaxies (Pettini et al 2000; 2001; 2002).

- The predicted wind velocity and outflow rate have no explicit dependence on the properties of dark halos which dominate the potential wells. Thus, galactic winds and mass outflows can occur in a variety of halos, provided that the cold gas density is high enough. This is in agreement with the observation that there is no strong correlation between wind properties and the total mass of a galaxy (Heckman et al 2000). The potential well of a galaxy will, however, determine whether the outflows escape from the galaxy or return.

- The fraction of galaxies with superwinds that will eventually escape from their dark matter haloes is a function of circular velocity and redshift. Our model predicts that the fraction is high in low circular velocity systems such as dwarf galaxies although their contribution to the total SFR is small. More interestingly, we find that the fraction is high for galaxies at high redshifts, such as the LBGs at redshift $z=3$. The winds will undoubtedly heat and chemically contaminate the IGM, and hence have important implications for subsequent galaxy formations in the preheated IGM.

We apply our model to make predictions for the properties of the winds expected from local starburst galaxies and high-redshift Lyman-break galaxies. These predictions can match many of the observed properties. We therefore believe that our model catches the main points required to model the superwind phenomenon. Our model is also simple, and so can be easily incorporated into numerical simulations and semi-analytical models of galaxy formation. 


\section{ACKNOWLEDGEMENT}

We thank Simon White for carefully reading the manuscript and useful suggestions. CS acknowledges the financial support of MPG for a visit to MPA. This project is partly supported by the Chinese National Natural Foundation and the NKBRSF G1999075406.

\section{REFERENCES}

Adelberger, K. L., Steidel, C. C., Shapley, A. E., Pettini, M., 2002, preprint (astro-ph/0210315)

Awaki, M., Ueno, S., Koyama, K., Tsuru, T., Iwasawa, K. 1996, PASJ, 48, 409

Clarke,C.J., Oey,M. S., 2002, preprint (astro-ph/0208442)

Dahlem, M., Weaver, K., Heckman, T. 1998, ApJS, 118, 401

Dawson, S., Spinrad, H., Stern, D., Dey, A., van Breugel, W., de Vries, W., Reuland, M., 2002, ApJ, 570, 9

Dekel A., Silk J., 1986, ApJ, 303, 39

Della Ceca, R., Griffiths, R., Heckman, T., MacKenty, J. 1996, ApJ, 469, 662

Della Ceca, R., Griffiths, R., Heckman, T. 1997, ApJ, 485, 581

Della Ceca, R., Griffiths, R., Heckman, T., Lehnert, M., Weaver, K. 1999, ApJ, 514, 772

Efstathiou G., 2000, MNRAS 317, 697 (E2000)

Ferrara A., Pettini M., Shchekinov Y., 2000, MNRAS, 319, 539

Forbes, D. A., Polehampton, E., Stevens, I. R., Brodie, J. P., Ward, M. J., 2000, MNRAS, 312 , 689

Furlanetto S. R., Loeb A., 2002, preprint(astro-ph/0211496)

Heckman, T. M., 2001, ASP Conf., 254, 292

Heckman, T. M., Lehnert, M. D., Strickland, D. K., Armus, L., 2000, ApJS, 129, 493

Heckman, T., Armus, L., Weaver, K., Wang, J. 1999, ApJ, 517, 130

Iwasawa, K., Comastri, A. 1998, MNRAS, 297, 1219

Martin, C. L., Kobulnicky, H. A., Heckman, T. M., 2002, ApJ, 574, 663

Katz N., Hernquist L., Weinberg D.H., 1999, ApJ 523, 463

Kauffmann G., Colberg J.M., Diaferio A. White S.D.M., 1999, MNRAS 303, 188

Kennicutt, Jr. R. C., 1998, ApJ, 498, 541

Lemson G., Kauffmann G., 1999, MNRAS 302, L111

Mac Low M., Ferrara A., 1999, ApJ, 513, 142

McKee C. F., Ostriker J. P., 1977, ApJ 218, 148 (MO77)

Mo H.J., Fukugita M., 1996, ApJ 467, L9

Mo H.J., Mao S., White S.D.M., 1998, MNRAS 295, 319

Mo H.J., Mao S., White S.D.M., 1999, MNRAS 304, 175

Mo H.J., Mao S., 2002, MNRAS 333, 768

Mo H.J., White S.D.M., 2002, MNRAS 336, 112

Olmi L., Testi L., 2002, A\&A, 392, 1053

Persic M., Salucci P., Stel F., 1996, MNRAS 281, 27

Pettini M., Samantha A. R., Steidel C. C., Adelberger K. L., Hunt M. P., Shapley A. E., 2002, ApJ, 569, 742

Pettini, M., Shapley, A. E., Steidel, C. C., Cuby, J., Dickinson, M., Moorwood, A. F. M., Adelberger, K. L., Giavalisco, M., 2001, ApJ, 554, 981

Pettini, M., Steidel, C. C., Adelberger, K. L., Dickinson, M., Giavalisco, M., 2000, ApJ, 528, 96

Scannapieco E., Ferrara A., Madau P., 2002, ApJ, 574, 590 
Scannapieco E., Ferrara A., Broadhurst T., 2000, ApJ, 536, L11

Shu C., 2000, A\&A 354, 815

Shu C., Mao S., Mo H. J., 2001, MNRAS, 327, 895

Silk J., 1997, ApJ, 481, 703

Silk J., 2001, MNRAS, 324, 313

Silk J., 2002, preprint(astro-ph/0212068)

Spitzer L. Jr. 1942, ApJ, 95, 329

Stark A. A., \& Brand J., 1989, ApJ, 339, 763

Steidel C. C., Pettini, M., Hamilton, D., 1995, AJ, 110, 2519

Strickland D. K., Heckman T. M., Weaver, K. A., Hoopes, C. G., Dahlem, M., 2002, ApJ, 568, 689

Strickland, D. K., Stevens, I. R., 2000, MNRAS, 314, 511

Suchkov A. A., Balsara D. S., Heckman T. M., Leitherner C., 1994, ApJ, 430, 511

Theuns, T., Viel, M., Kay, S., Schaye, J., Carswell, R. F., Tzanavaris, P., 2002, ApJ, 578, L5

Tomisaka K., Bregman J. N., 1993, PASJ, 45, 513

Warren M. S., Quinn P. J., Salmon J. K., Zurek W. H., 1992, ApJ 509, 19

White S.D.M., Frenk C.S., 1991, ApJ, 379, 52

White, M., Hernquist L., Springel V., 2002, ApJ, 579, 16 